UCRL-JC-107423

PREPRINT

\title{
Benchmark Field Study of Deep Neutron Penetration
}

\author{
James F. Morgan and Kenneth Sale \\ Lawrence Livermore National Laboratory \\ P.O. Box 808, Livermore, CA 94550
Raymond Gold, James H. Robert, and Christopher C. Preston Metrology Control Corporation Richland, WA 99352

This paper was prepared for submittal to The Proceedings of the Seventh ASTM-EURATOM - -

Symposium on Reactor Dosimetry

Strasbourg, France

August 27-31, 1990

hutivin boly

JUL 291991

June 10, 1991

$\lambda$

This is a preprintof apaperintended forpublieation in a joumal or proceed inga. Since changes may be made before publication, this preprint is made available with the understanding that it will not be cited or reproduced without the permiesion of the author.

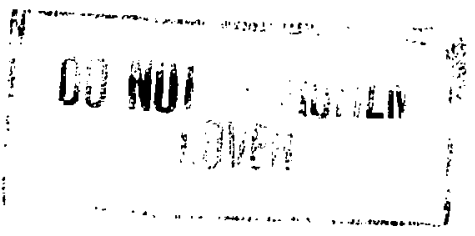

\section{MASTER}




\section{DISCLAIMER}

This document was prepered as an eccougl of mork sponsored by an ageacy of the United States Goxcrameat. Nesher the Unfied Sthtes Goyesnmeat bor the University of Californts nor asy of thetr employeses, makes any marcanty. express or implied, or essugres any legal liability or reponsibllity for the acearacy, complestessh of unefof. ness of any iatoratelon, appentus, produet, or prosess diselosed, or pepresents that its use would not Infriage privately orned alghts. Reterence herein to any speeitc cominercial products, process, or service by vade name, indevark, manufarturer, or otherwlse. does not pecessarily constitule or imply its endorcement recommeadallon.

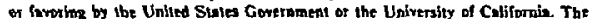
views and opinions of authors expressed berefa da col necessorily saste or reflect those of the Uniled Sites Govemriebt or the Univerity of Calltorain and shall not be used for advertising ar product endorsement purposes. 


\title{
Benchmark Field Study of Deep Neutron Penetration \\ James F. Morgan and Kenneth Sale \\ Lawrence Livermore National Laboratory \\ P. O. Box 808, Livermone, CA 94550 \\ UCRL $-J C--107423$ \\ DE91 015677 \\ Raymond Gold, James H. Roberts and Christopher C. Preston \\ Metrology Control Corporation \\ Richland, Washington 99352
}

\begin{abstract}
A unique benchmark neutron field has been established at the Lawrence Livermore National Laboratory (LLNL) to study deep penetration neutron transport. At LLNL, a tandem accelerator is used 10 generate a monoenergetic neutron source that permits investigation of deep neutron penetration under conditions that are virtually ideal to model, namely the transport of mono-nergetic neutrons through a single material in a simple geometry. General features of the Lawrence Tandem (LATAN) benchmark field are described with emphasis on neutron source characteristics and room return background. The single material chrosen for the first benchmark, LATAN-1, is a steel repre-

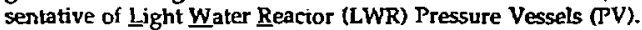

\section{Introduction}

In recognition of significant safety and economic issues associated with radiation-induced embrittlement of reactor pressure vessels, the U. S. Nuclear Regulatory Commission (NRC) sponsored the light water reactor pressure yessel surveillance dosimetry improvement program (LWR-PV-SDIP) over the last decade or more to improve, maintain, and standardize neutron dosimetry, damage correlation, and the associated reactor analysis procedures used to predict the integrated effect of neutron exposure to LWR.PV. Owing to the complexity of this embrittlement phenomenon, experimental and calculational strategies formulated in the LWR-PV-SDIP wene subsequently implemented in a multi-laboratory international program.

Four principal laboratories collaborated in the conduct of this program:

Centre D'Etude De L'Energie Nucleaire/Studiecentrum Voor Kernenergie (CEN/SCK), Mol, Belgium;

Hanford Engineering Development Laboratory (HEDL), Richland, Washington;

National Bureau of Standards (NBS), Gaithersburg, Maryland;

Oak Ridge National Laboratory (ORNL), Oak Ridge, Tennessee.

-This work was performed under the auspices of the U. S. Dept. of Energy by the Lawrence Livermore National Laboratory. 
Subsequently, collaboration with additional European laboratories was established:

Rolls-Royce \& Associates, Ltd., (RRA), Derby, U. K

United Kingdom Atomic Energy Establishment Winfrith (UKAEEW), Winfrith, U.K.

Kernforschungsanlage, Julich, GmbH, (KFA), Julich, Federal Republic of Germany.

Through the complementary capabilities of personnel at these seven lahoratories, it has been possible to carry out a productive and highly cost-effective prosiam. The major benefit of LWR-PV-SDIP has been and continues to be a significant improvement in the accuracy of the assessment of the current metallurgical condition and the remaining safe operating lifetime of LWR-PV. 1 These efforts have been extensively reported in the ASTM-EURATOM series of symposia on Reactor Dosimetry, and a review of the status and accomplishments of the LWR-PV-SDIP was presented at the very last symposia in Jackson Hole. ${ }^{2}$

Benchmark fields have served as important elements in implementing experimental and calculational strategies adopted in the LWR-PV-SDIP. In the broadest sense, a benchmark neutron field represents a practical test bed for the testing, vaiidation, and confrontation of different methods, be they experimental or theoretical. Progress by trial and error is, perhaps, the essence of benchmark field work. One essential characteristic of a benchmark field is that all environmental variables are well controlled and accurately defined. Benchmark fields can be used primarily for calculational purposes. In many instances they are used for both experimential and calculational purposes simultancously.

Independent contributions from U.S. and EURATOM laboratories participating in LWR-PV-SDIP benchmark field studies have confirmed the existenve of a serious and persistent discrepancy between neutron field calculations and experiment. The calculated-to-experimental $(C / E)$ ratio is considerably less than unity in LWR-PV environments. Moreover, this discrepancy increases, i.e., C/E decreases farther from unity, with increasing neutron energy and with increasing depth of penetration into the PV.

This discrepancy is the most significant issue in current LWR-PVS neutron dosimetry efforts. Since the PV is non-replaceable, radiation induced embrittlement of the PV controls the effective lifetime of a nuclear power plant. Because of the critical nature of the PV, successful resolution of this discrepancy would have a major impact on current plant life extension (PLEX) efforts for LWR.

It must be emphasized that the same general discrepancy has arisen in another international neutron dosimetry program conducted for completeiy different reasons. Within nearly the same time frame that LWR-PV-SDIP was conducted, a joint US-Japan program was carried out to re-evaluate radiation exposures of atom bomb survivors. A final report describing the results and ce nclusions of this program, which has been designated by Dosimetry System 1986 (DS86), has been issued. ${ }^{3}$ In general, studies of Hiroshima and Nagasaki atom bomb survivors have provided valuable data for quantifying the effects of radiation on man. These data have been used by the United Nations Scientific Committee on the Effects of Alomic Radiation (UNSCEAR) for comprehensive assessments of the effects and risks of jonizing radiation. Conclusions of UNSCEAR reports 4,5 often form the basis that governments use for policy decirions on radiation protection standards.

An essential part of this joint U.S.-Japan re-assessment program for evaluating radiation doses at Hiroshima and Nagasaki has been the replication of Little Boy in the form of a crilical reactor assembly. Replication of Littla Boy which was the atom bomb exploded at Hiroshima, was faithfully accomplished at Los Alamos National Laboratory (LANL). Avoilable specifications together with 
spare or excess parts were used to set up and operate the Little Boy replica as a low power critical reactor assembly at LANL. Replication of Lilfle Boy included the thick steel casing of the bomb. The thickness of this casing varied with polar angle, with a minimum thickness at the waist of the assembly $\left(90^{\circ}\right)$, which is comparable to the nominal 8-inch thickness of a LWR-PV. The steel casing thickness increases with decreasing polar angle to a maximum thiskness near the top of the assembly $\left(0^{\circ}\right)$ that is considerably larger than the nominal LWR-PV thickness. Consequently, the Little Boy replica shares one significant feature with all LWR-PV-SDIP benchmarks, namely deep penetration neutron transport in steel.

In view of the sparsity of neutron dosimetry data that actually exists from the Hiroshima and Nagasaki sites, 6 calculations have been relied upon to generate A-bomb radiation exposures. Hence, this Little_Boy replica constitutes a benchmark radiation field for the testing and intercomparisons of experimental and calculational radiation metrology methods. Rigorous testing of these calculational methods using the Little Boy cenchmark was a crucial aspect of this program. Extensive experimental and calculational efforts have heen expended to characterize the radiation field emitted by the Little Boy replica. ${ }^{7-9}$ Both the neutron and gamma-ray components of this mixed radiation field have been addressed. These Little Boy replica characterization efforts are reviewed in Chapter 2 of the final report on DS86, 10 where it is concluded that the C/E ratio is acceptable at the waist of the critical assembly $\left(90^{\circ}\right)$, but $\mathrm{C} / \mathrm{E}$ decreases and can be as low as 0.7 at the top of the critical assembly $\left(0^{\circ}\right)$.

This $\mathrm{C} / \mathrm{E}$ trend is evident in nuclear research emulsion (NRE) neutron spectrometry carried out at the Little Boy replica. ${ }^{11,12}$ However, the more detailed absolute power normalization experiments of the Little Boy replica conducted with solid state recorders (SSTR) ${ }^{13}$ yield a C/E behavior for these NRE data that is qualitatively identical to LWR-PV-SDIP benchrnark results. More specifically, one finds that $\mathrm{C} / E$ decreases with increasing penetration from approximately 0.7 at $90^{\circ}$ to 0.5 at $0^{\circ}$. Moreover, these NRE data also confirm the trend for C/E to decrease with increasing neutron energy.

\section{Speculative Ad-Hoc Solutions}

The persistent nature of this discrepancy in different LWR-PV-SDIP benchmark fields, such as the PCA, VENUS and NESDIP, has fueled the speculation that deficiencies exist in the neutron cross section of iron. In fact, two different groups have introduced different adjustments of the inelastic cross section of iron to obtain improved C/E ratios. ${ }^{14-17}$ However, these adjusted iron cross sections do not provide consistent improvements, but rather results that vary from one LWR-PV environment to another.

Concern about the uniqueness of such cross section adjustments has already been raised. 2 Indeed, arbitrary changes in the iron inelastic cross section can reduce the magnitude of this discrepancy for certain cases, but there is no definitive experimental basis for making these adjustments. It is, therefore, not surprising to learn that this (non-unique) adjus!ment does not consistently provide unproved C/E ratios in other environments.

Perhaps an even simpler ad-hoc solution can be obtained by adjusting the high energy portion of the fission neutron spectrum. Indeed, the physics of deep penetration transport in reactor environments places considerable importance on the high energy portion of the fission spectrum. Moreover, for deep penetration neutron transport, emphasis is not placed on a fixed energy region of the fission neutron spectrum, but rather shifts upward in energy as the depth of penetration increases. Deficiencies in this high energy region, in particular use of a source term that provides too few high energy neutrons, could qualitatively explain both the energy and penetration dependent trends of the discrepancy obtained in the LWP.PV-SDIP and the Litkle Boy benchmarks.

Examination of the experimental data defining the t: gher c nergy region of the fission neutron spectrum, especially above $8 \mathrm{MeV}$, revals very poor statistical precision. Moreover, the latest comparison 
between theory and experiment ${ }^{18}$ suggests significant uncertainties in this high energy region of the fission neutron spectrum. However, arbitrary adjustments of the high energy region of this spectrum can yield solutions that possess the same non-qniqueness obtained from cross section adjustments.

\section{Deep Penetration Neutron Benchmark Field}

Benchmark neutron fields established for both LWR-PV-SDIP and Little Boy have addressed specific real world issues. In so doing, these benchmarks have had to simulate the actual transport of fission spectrum neutrons through composite materials in complex geometries. While these elements were necessary to the basic purpose of these benchmarks, they present serious difficulties for the resolution of the observed discrepancy.

Indeed, it is difficult, if not impossible, to resolve this significant discrepancy in neutron dosimetry let alone attain more stringert accuracy goals required to implement PLEX or establish personnel radiation protection standards with a "business as usual" approach. Use of the same experimental and theoretical methods, whether in power reactor PV environments or LWR-PV benchnark field tests, will in all likelihood repeat past history rather than provide new insights. Results from the series of dedicated LWR-PV benchmarks at VENUS and NESDIP verify this conclusion. What ts needed is a new. and independent benchmark field that provides simpler and more ideal conditions for the comparison of theory and experiment, so that a higher plane of understanding of deep penetration neutron transport can be achieved.

To satisfy this need for a higher piane of understanding, a unique benchmark field has been set up at the Lawerence Livermore National Laboratory (LLNL). At LLNL, a tandem accelerator facility is used to study neutron transport from a monoenergetic source through a single material in a simple geometry.

Some of the more significant advantages of the Lawrence Tandem (LATAN) benchunark field are:

1. Simplified Geometry
(a) Single Material
(b) Poirtt Solirce
(c) Cylindrical Symmetry

2. Monoenergetic Neutron Source

(a) Energy of the monoenergetic source neutrons can be varied

(b) Independence of the fission neutron spectrum

The single material chosen for the first benchmark, LATAN-1, is a steel representative of LWR$P V$. Becausc it has been demonstrated that active neutron detectors, such as fission chambirs and proportional counters, produce non-negligible perturbations, measurements in LATAN-1 will initially emphasize passive dosimeters. Passive neutron dosimeters of high sensitivity and absolute accuracy will be used, such as SSTR and NRE. In contrast with conducting a sequence or series of irradiations, LATAN-1 irradiations have used a three-dimensional array of these passive dosimeters embedded at different mean-frec-path locations from the point source. This approach reduces the ieed for normalization between irradiations with a concommitant reduction of systematic uncertainty. 


\section{The LATAN Benchmark Neutron Field}

Monoenergetic neutrons for the irradiations are generated by nuclear reactions in a gas target syss tem. The deuteron beam from the LLNL Tandem is incident on a newly designed gas cell locaied at the end of the 0 beam line. By filling the cell with various gases, such as deuterium or tritium, monoenergetic neutrons are producer with energies from 2 to $20 \mathrm{MeV} .19$ For a typical gas fill of 5 atm of deuterium, a neutron beam of $5 \times 10^{7}$ neutrons/sec, strongly peaked around zero degrees, is produced. For LATAN-1, these neutrons are then incident on the center of a cylindrically symmetric assembly of the representative steel with appropriately placed neutron dosimeters, such as SSTR, located throughout the assembly. The absolute magnitude of the neutron flux is measured by the associated particle technique. A solid state detector is used to count the number of charged particles produced in the gas cell and this result is used to determine the absolute number of emitted neutrons.

One of the most important limitations of this kind of benchmark neutron field can be the room netum background, i.e., the neutrons which scatter in the matf,cial surrounding the experimental facility and then find their way back into the assembly. The experimental hall at the LLNL Tandem was designed to reduce this problm. The walls are thin and most of the neutrons are allowed to pass out of the experimental area and to interact at a large distance in an earthen berm, where their effect is reduced by $1 / R^{2}$. However, the floor of the experimental hall is heavy concrete, and the hall contains heavy experimental equipment such as switching and quadrupoles magnets, which can act as effective neutron scattering sources. Conseguently, a combination of experimental and computational methods are used to Lnderstand the magnitude of this room retum effect.

Experimentally the magnitude $-f$ the room retum can be studied by using a pulsed deuteron beam and active neutron detectors. A short pulse $(-1 \mathrm{~ns})$ of deuterons is incident on the gas cell and active neutron detectors located within the assembly are used to measure the return neutron intensity as a function of time. These results are compared with extensive Monte Carlo neutron transport calculations, which mock-up most of the significant neutron scattering sources. The agreement between measured and calculated values of the room return increases our confidence in the values assigned to this background effect. 


\section{REFERENCES}

1. R. Gold and W. N. McElroy, Radiation-Induced Embrittlement in Light Water Reactor Pressure Vessels, Nucl. Eng. and Design 104, 155-174 (1987).

2. R. Gold and W. N. McElroy, The light water pressure vessel surveillance dosimetry improvernent program (LWR-PV-SDIP): Past accomplishments, recent developments and future directions, in: Proc. of the Sixth ASTM-EUTRATOM Symposium on Reactor Dosimetry, June 1-5, 198?, Jackson Hole, WY, STP 1001, 44-61 (1989).

3. Radiation Effects Research Foundation. Dosimetry system 1986, U. S.-Japan joint reassessment of atomic bomb radiation dosimetry in Hiroshima and Nagasaki. Hiroshima, Japan: Raciation Effects Research Foundation, 1987.

4. United Nations Scientific Committee on the Effects of Atomic Radiation. Genetic and somatic effects of ionizing radiation. 1986 report to the General Assembly, with annexes. New York: United Nations, 1986.

5. United Nations Scientific Committee on the Effects of Atomic Radjation. Sources and effects of ionjzing radiation. 1988 report to the General Assembly, with annexes. New York: United Nations, 1988.

6. W. E. Loewe, E. Mendelsohn, T. Hamada, T. Maruyama, S. Okajim, J. V. III Pace, M. Sakanoue, S. Kondo, T. Hashizume, J. Marcum, and W. A. Woolson, "Measurements of Neutron Fluences," Chapter 5 in U.S.-Japan Ioint Reassessment of Atomic Bamb Radiotion Dosimetry in Hiroshima and Nagasaki, Final Report, Vol. 1, Radiation Effects Research Foundation, 5-2 Hijiyama Park, Minar.iKu, Hiroshima, 732, Japan (1987).

7. "Little Boy Replica, A Seminar to Discuss Construction, Operation, Measurements and Calculation, Los Alanos National Laboratory, Los Alamos, NM, September, 1983.

8. Measurements on a True Replica of Lillle Boy Special Session 8, Computer Applications in Health Physics, Proceedings of the 17 th Midyear Topical Sympssium of the Health Physics Society, Pasco, WA, February, 1984.

9. Replication of Little Boy, Special Sessions I and II, 29th Annual Meeting of the Health Physics Society, New Orleans, LA, June, 1984.

10. P. P. Wholen, "Calculation and Verification of Source Terms," Chapter 2 in U. S.-Japan Joint Reassessment of Atomic Bomb Radiation Dosimetry in Hiroshima and Nagasaki. Final Report, Vol. 1, Radiation Effects Research Foundation, 5-2 Hijiyarra Park, Minami-ku, Hiroshima, 732, Japan (1987).

11. R. Gold, J. H. Roberts, and C. C. Preston, INuclear Research Emusion Neutron Spectrometry at the Little Boy Replica, HEDL-7559, October, 1985.

i2. J. H. Roberts, R. Gold, and C. C. Preston, Measurements of the Absolute Neutron Fluence Spectrum Emitied al $0^{\circ}$ and $90^{\circ}$ from the Lillle Boy Replica, 13th International Conference on Solid State Nuciear Track Delectors, Rome, Seplember 23-27, 1985, Nucl. Track and Radiation Measurements 12, 553 (1986).

13. R. Gold, ). H. Roberts, and C. C. Preston, Little Boy Replica Pozer Nurmalization, 34th Annual Meeting of the Health Physics Society, Albuquerque, June 25-29, 1959. 
14. J. Buter, M. D. Carter, E. J. Curl, M. R. March, A. K. MoCracken, M. F. Murphy, and A. Packwood, The PCA Replica Experiment: Part I, Winfrith Medsurements and Calibrations, NUREG/CR-3324. AEEW-R 1736, Part I, NRC, Washington, D. C, January, 1984.

15. M. D. Carter, et al., The ASPIS Iron Benchmark Experiment: Results and Calculational Model, in Proceedings, OECD Specialists' Meeting on Nuclear Data and Benchmarks for Shielding, Paris, 1980.

16. R. E. Maerker, LEPRICON Amalysis of Pressure Vessel Surveillance Dosimetry Inserted into H. B. Robinson-2 during Cycle 9, NUREG/CR-4439, ORNL/TM-1032, 1986.

17. R. E. Maerker, Application of LEPRICON Methodology to LWR Pressure Vessel Suroeillance Dosimetry, Sixth ASTM-EURATOM Symposium on Reactor Dosimetry, June 1-5, 1987, Jackson Hole, WY, STP 1001, 405-414 (1989).

18. D. Madland, and J. Rayford Nix, New Calculations of Prompt Fission Neutron Spectra and ..., LANL REPORT LA-UR-81-2968.

19. J. E. Brolly and J. L. Fowler, "Monoenergetic Neutron Sources: Reactions with Light Nuclei" in Fast Neutron Physics, Part 1. J. B. Marion \& J. L. Fowler, ed., Interscience Publishers, Inc., London, 1960. 